\title{
A Comparative Phonocardiography Study: Two Wavelet Based Methods for Fetal Heart Sound Detection
}

\author{
Elisavet Koutsiana ${ }^{1}$, Leontios Hadjileontiadis ${ }^{2,3}$, Ahsan H Khandoker ${ }^{3,4}$, Ioanna Chouvarda ${ }^{1}$ \\ ${ }^{1}$ Laboratory of Medical Informatics, the Medical School, Aristotle University, Thessaloniki, Greece \\ ${ }^{2}$ Department of Electrical \& Computer Engineering Aristotle University, Thessaloniki, Greece \\ ${ }^{3}$ Department of Electrical \& Computer Engineering, Khalifa University of Science and Technology, Abu \\ Dhabi, UAE \\ ${ }^{4}$ Department of Biomedical Engineering, Khalifa University of Science and Technology, Abu Dhabi, UAE
}

\begin{abstract}
The Fetal Heart Rate (FHR) periodic monitoring is an essential part during pregnancy. Auscultation is a noninvasive low cost technique for the heart sound examination. However, Fetal Phonocardiogram (fPCG) signals are correlated with high background noise due to the maternal organs. The aim of this study is to develop an automatic analysis technique to identify the location of the FHSs in fPCGs signals and calculate the FHR. In this paper two denoising methods are proposed and compared in real and simulated fPCGs signals. The signals were analyzed in the Wavelet domain and then two methods were proposed for the detection of the FHSs. The first approach combines the Wavelet Transform with the Fractal Dimension analysis (WT-FD) while the second approach combines the Wavelet Transform with Simplicity Filtering (WT-S), which is computed by the eigenvalue spectrum method. Both methods present good performance with the WT-FD presenting $88 \%$ precision, $93 \%$ recall and WT-S $71 \%$ and $72 \%$ respectively in fetal heart sounds detection in simulated signals from -4.4 to $26.7 d B$, while the WT-S method is more unstable in cases with robust noise than the WT-FD.For the real data preliminary results are presenting and analyzed.
\end{abstract}

\section{Introduction}

In the early 1960s Electronic Fetal Monitoring (EFM) was presented as a new, developed tool for the fetal examination during pregnancy and childbirth [1]. Some common EFM devices are the doppler ultrasound, the cardiotocogram, the fetal electrocardiogram, the fetal phonocardiogram and the fetal cardiac MRI.

One of the most important parameters for fetal health examination during pregnancy is the Fetal Heart Rate (FHR) monitoring through which it is possible for the clinician to examine the fetal growth and physiology. Although, the doppler ultrasound is the typical device for examination of the FHR, this technique cannot be used for long-term monitoring mainly because of the cost of the monitoring device, the complexity of its use and the long-term exposure to ultrasound energy [2].

A passive alternative for long term monitoring of the fetus is the fetal heart auscultation. Auscultation is a lowcost and non-invasive method as it captures the acoustic signal of the Fetal Heart Sounds (fHSs) from the mother's abdominal surface [3]. The acoustic signal produced by the fHSs can be visually depicted in the Fetal Phonocardiogram (fPCG).

Nevertheless, fetal auscultation has many challenges because the fPCG signals are contaminated with noise from various sources, such as maternal heart sounds, digestive sounds, maternal respiration movements, fetal movements, external noise and others [3].

In our previous studies, we have proposed the Wavelet Transform - Fractal Dimension (WT-FD) method for FHS detection in fPCG signals [4]. In this paper, the Wavelet Transform - Simplicity (WT-S) method is suggested and compared with the WT-FD in PPCG signals.

Through the years, many studies were proposing the WT combined with the S analysis for the heart sound segmentation. A WT-S based method was proposed by the D.Kumar et al. in [5] for heart murmur segmentation while an Energy based and S based segmentation method for features computed from multi-level Wavelet decomposition coefficients was proposed by J.Vepa et al in [6]. Moreover J.Korver presented a study about the comparison of WT-S based heart sound and murmur segmentation methods in [7].

The aim of this study is to evaluate the performance of the two proposed methods in order to develop an automatic analysis technique to identify the location of the FHSs in fPCGs signals and calculate the FHR.

Figure 1 shows a flow chart for the two proposed methods. The normalized input signal $x(t), t=1, \ldots, N$ is decomposed into wavelet levels $W T_{i}(t), i=1, \ldots, M$ based on the signals' length and a level selection is applied. After that, one of the preferred FD based or S based analysis is performed in the selected wavelet coefficients to create the estimated sequence. Then, the Peak Peeling Algorithm (PPA) is used to gather only the peaks that represent heart sounds. A binary sequence segments the 
FHSs from the background noise and the reconstruction of the signal is achieved. The whole procedure iteratively continues until the stopping criterion is satisfied.

The paper is structured in three main sections: the first section briefly describes the mathematical background of the proposed methods, in the second section the results of the research are described and finally the third section completes the paper with conclusion.

\section{Mathematical Background}

After preprocessing, where needed, the proposed iterative method includes multiple steps, as depicted in Figure 1. The steps are described in more detail in the following subsections.

\subsection{Wavelet Transform}

In this study, the fPCG signals were processed by means of stationary Wavelet Transform (WT), which is a time-frequency analysis method. Wavelets are families of functions generated from a single base wavelet called the 'mother wavelet', by dilations and translations [8]:

$$
\psi_{\alpha, b}(t)=\frac{1}{\sqrt{\alpha}} \psi\left(\frac{t-b}{\alpha}\right), \alpha>0, b \in R
$$

where $\alpha$ is the dilation parameter and $b$ is the translation parameter.

The Daubechies family of wavelets was chosen to decompose the fPCG signals into levels. After the wavelet decomposition, an energy based selection is performed for the subtraction of the first noisy levels.

\subsection{Fractal Dimension}

The Fractal Dimension (FD) is a tool that reflects the signal complexity in the time domain. In this research FD was adopted as a means to detect the FHSs in the WT domain [8]. The Katz FD definition is estimated by:

$$
F D=\frac{\log _{10}(n)}{\log _{10}\left(\frac{d}{L_{C}}\right)+\log _{10}(n)},
$$

where $L_{C}$ is the total length of the curve, realized as the sum of distances between successive points, $d$ is the distance between the first point of the sequence and the point of the sequence that provides the farthest distance and $n=L_{C} / a$ is the number of steps in the curve, where a denotes the average step, i.e., the average distance between successive points.

The FD technique is performed using a sliding window of $W=\operatorname{int}(0.05 F s)$ samples length in each selected WT level, where int(.) indicates the integer part of the argument, the constant is empirically set at 0.05 and $F s$ denotes the sampling frequency of the signal.

\subsection{Simplicity}

Simplicity and especially the eigenvalue spectrum is another means to measure the complexity of a fPCG signal. The background noise and murmurs in PCG signals are characterized as high complex components while heart sounds as less complex components of the signals [5, 7]. Low complexity implies high simplicity making the heart sounds separate from the background noise.

Let $x(t)$ be the input signal and the delay vector $x_{i}=[x(t), x(t-\tau), \ldots, x(t-(m-t) \tau)]^{T}$ where $T$ symbols the transpose. The embedding matrix is defined as:

$$
X=\frac{1}{\sqrt{P}}\left[x_{1}, x_{2}, \ldots, x_{P}\right]
$$

and $\tau$ is the embedding delay, $\mathrm{m}$ is the embedding dimension and $P=N-(m-1)$. The correlation matrix is $C=X^{T} X$.

Let $D=\left\{\lambda_{1}, \lambda_{2}, \ldots, \lambda_{m}\right\}$ be the singular spectrum of the embedding matrix $X$ i.e. the diagonal matrix with the eigenvalues of $C$ sorted in descending order. Let $H$ be the entropy of the calculated normalized eigenvalues $\hat{\lambda}_{k}^{i}$ :

$$
H(i)=\sum_{k=1}^{m} \hat{\lambda}_{k}^{i} \log \hat{\lambda}_{k}^{i}
$$

The complexity can be calculated by $\Omega^{i}=2^{H(i)}$ while the simplicity by $S^{i}=1 / \Omega^{i}$.

\subsection{Peak Pealing}

Through a self-adjusted iterative procedure, the PPA iteratively 'peels' the estimated sequences in order to automatically detect the peaks resulting in the estimated FD or Simplicity sequence [8]. The iteration procedure starts with a threshold operation based on the mean value and the standard deviation of the vectors as follows:

$$
P P A_{j}=\left\{\begin{array}{l}
F D_{j}^{z}, F D_{j}^{z}>\mu^{z}+\sigma^{z} \\
1.0, \text { elsewhere }
\end{array}, j=1, \ldots, M\right.
$$

where $\mu^{z}$ denotes the mean value of the vector, $\sigma^{z}$ is the standard deviation of the vector and $z$ is the number of the self-adjusted iterations. The iterative procedure stops when the mean square error criterion is satisfied. A flow chart of the PPA procedure with details is available in [9].

The PPA method applied to the $F D_{j}$ or $S_{j}$ sequence leads to two binary sequences for each wavelet coefficient, one for the FHSs segmentation $S B T H_{j}(t)$ and one for the background noise extraction $N B T H_{j}(t)$. After the reconstruction of the two subsets the algorithm decides based on the mean square error if the procedure stops or if there is more information in the background noise about the sounds and the whole procedure iterates. 


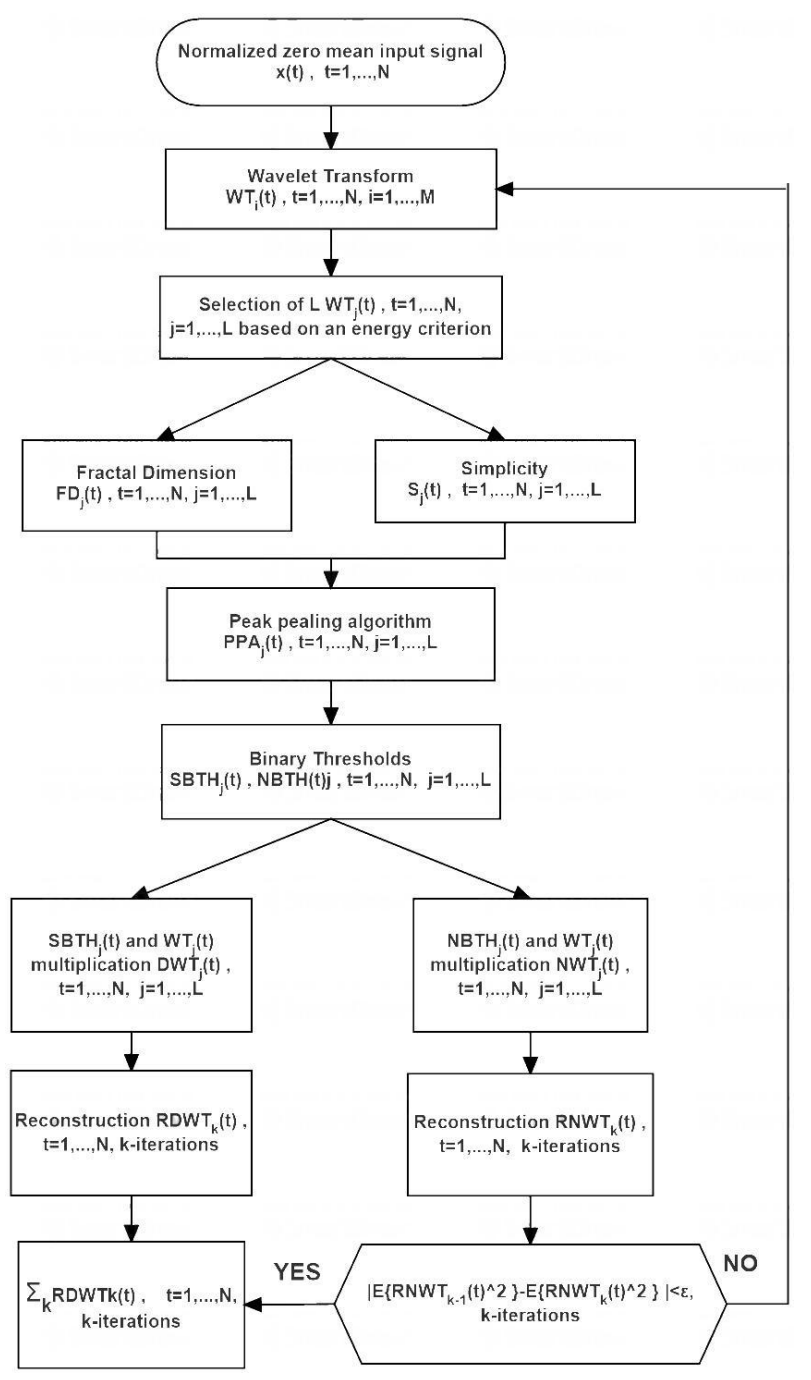

Figure 1. A shementic representation of the iterative procedure of the two methods.

\section{Results}

The analysis of this study was applied on a personal computer using Matlab R2015a and tested on simulated data and on three real signals. Every input signal was tested for 10sec for the simulated signals and for $60 \mathrm{sec}$ for the real signals considering $\mathrm{Fs}=1000 \mathrm{~Hz}$.

A Simulated Web database from Physionet named simfpcgdb was used for the comparison of the two methods (https://physionet.org/pn3/simfpcgdb/). The simulated database created by Cesarelli et al. [3]. Simulated fPCG signals were generated including simulated S1 and S2 sounds, corrupted by noise. These signals are qualified by a range of SNR values which were computed in $\mathrm{dB}$.

The two compared methods were also tested in Real fPCG signals from a small pilot study. The fPCG signals were recorded using 4 vibration sensors [9].

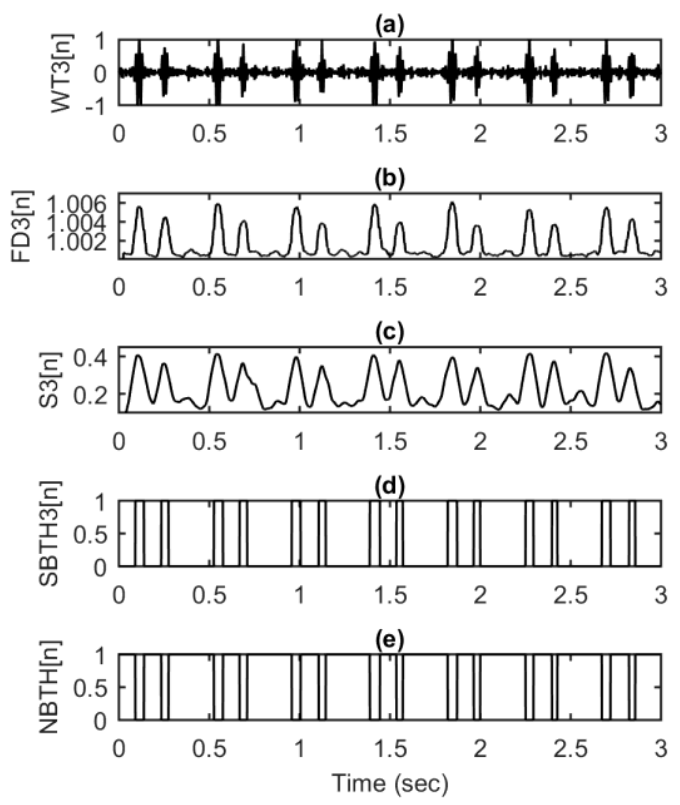

Figure 2. (a) Wavelet Transform third level coefficient, (b) Fractal Dimension sequence, (c) Simplicity sequence, (d) Binary thresholded components related to the heart sounds, (e) Binary thresholded background noise.

For the evaluation of the two methods in the simulated signals the Recall (R), the Precision (P) and the geometric mean of theratios of true positive to recorded sounds and true positive to the detected sounds (GM) are calculated. The simulated signals were divided in three groups based on their Signal to Noise Ratio (SNR) and the mean results are shown in Table 1 and Table 2.

A second criterion for the evaluation of the methods was the fHR calculation. The locationsof the S1 heart sounds were identified by applying peak to peak time distance and amplitude criteria. The actual fHR of the simulated signals was $144 \mathrm{bpm}$ and the methods results are shown in Table 1.

In both cases the methods successfully detect the location of the fHS with good performance in signals with high SNR while the WT-FD method presents better performance than the WT-S method in cases of low SNR.Based on the R index in Table 2 the WT-S method detects just over half of sounds in the last group of signals and based onthe $\mathrm{P}$ index approximately half of the detected as soundsare false positive. However, compared the results in Table 1 and Table 2 it appears that almost all ofthe correct predictive sounds are S1 heart sounds and the fHR calculation is close to the actual rate. Hence, the method is not efficient in S2 heart sound detection in cases with low SNR. In real fPCGs signals a preprocessing filter with the combination of the four different channels is a necessary additional step to remove the sounds of the mother in order to reveal and study the 
Table 1. Results of S1 heart sound identification in simulated data for the two compared methods and fHR calculation.

\begin{tabular}{ccccccccc}
\hline \multirow{2}{*}{ SNR range } & \multicolumn{4}{c}{ WT-FD } & \multicolumn{4}{c}{ WT-S } \\
\cline { 2 - 9 } & $\mathrm{R}$ & $\mathrm{P}$ & GM\% & fHR bpm & $\mathrm{R}$ & $\mathrm{P}$ & GM\% & fHR bpm \\
\hline$[-4,-18]$ & $0.99 \pm 0.03$ & $1.00 \pm 0.02$ & $99.1 \pm 2.39$ & $140.2 \pm 0.86$ & $0.98 \pm 0.05$ & $1.00 \pm 0.00$ & $98.9 \pm 2.72$ & $140.4 \pm 0.2$ \\
{$[-19,-23.1]$} & $0.99 \pm 0.02$ & $1.00 \pm 0.00$ & $99.6 \pm 1.04$ & $140.5 \pm 0.09$ & $0.97 \pm 0.05$ & $1.00 \pm 0.00$ & $98.4 \pm 2.43$ & $140.3 \pm 0.19$ \\
{$[-23.3,-27]$} & $0.98 \pm 0.03$ & $1.00 \pm 0.00$ & $99.1 \pm 1.3$ & $140.6 \pm 0.15$ & $0.93 \pm 0.06$ & $1.00 \pm 0.00$ & $96.4 \pm 2.9$ & $140.4 \pm 0.32$ \\
\hline
\end{tabular}

Table 2. Results of S1 and S2 heart sound identification in simulated data for the two compared methods.

\begin{tabular}{ccccccc}
\hline \multirow{2}{*}{ SNR range } & \multicolumn{3}{c}{ WT-FD } & \multicolumn{3}{c}{ WT-S } \\
\cline { 2 - 6 } & $\mathrm{R}$ & $\mathrm{P}$ & $\mathrm{GM} \%$ & $\mathrm{R}$ & $\mathrm{P}$ & $\mathrm{GM} \%$ \\
\hline$[-4,-18]$ & $0.98 \pm 0.03$ & $0.93 \pm 0.12$ & $94.30 \pm 7.8$ & $0.87 \pm 0.17$ & $0.82 \pm 0.13$ & $82.76 \pm 14.4$ \\
{$[-19,-23.1]$} & $0.93 \pm 0.06$ & $0.92 \pm 0.13$ & $91.39 \pm 10.5$ & $0.70 \pm 0.19$ & $0.72 \pm 0.14$ & $68.88 \pm 16.1$ \\
{$[-23.3,-27]$} & $0.88 \pm 0.03$ & $0.79 \pm 0.05$ & $81.79 \pm 4.9$ & $0.61 \pm 0.04$ & $0.60 \pm 0.05$ & $57.83 \pm 4.41$ \\
\hline
\end{tabular}

fHSs. In real recordings neither method identifies all the sounds contained in every signal, but they detect a sufficient number to make a fHR approach. Table 3 shows the comparison, of $1 \mathrm{~min}$ analysis, of the two methods with simultaneous electrocardiogram recordings. It shows that in normal fHR, like in patient 1 , both methods identify successfully the fHR with the WT-S presenting more unstable performance than the WT-FD. Patients 2 and 3 presents lightly slow or fast fHR respectively, with the mother patient 2 having higher HR than the fetal. In these cases both methods demonstrate low performance needing additional steps to complete this failing. Preliminary results indicatethat it is worth analyzing the application of the two methods to real data in a more extensive study.

Table 3. Results of fHR calculation in real data for the two compared methods WT-FD and WT-S.

\begin{tabular}{cccc}
\hline \multirow{3}{*}{ Patient } & \multicolumn{2}{c}{ PCG } & ECG \\
& \multicolumn{2}{c}{ fHR bpm } & fHR bpm \\
\cline { 2 - 4 } & WT-FD & WT-S & \\
\hline 1 & $147 \pm 6.7$ & $150 \pm 46.2$ & $152 \pm 2.5$ \\
2 & $139 \pm 2.6$ & $127 \pm 12.6$ & $109 \pm 1.7$ \\
3 & $167 \pm 6.1$ & $147 \pm 39.8$ & $203 \pm 0.0$ \\
\hline
\end{tabular}

\section{Conclusion}

The aim of this study was to compare two methods applying in fPCG signals and test their performance relatively to the SNR intensity. The research indicates that both methods approach differently the heart sounds compared to the type of noise containing in the signals, adding a new perspective for better segmentation of fHS from the different types of maternal sounds.

\section{Acknowledgements}

The real fPCG data were obtained from a research project funded by Abu Dhabi Education Council (ADEC).

\section{References}

[1] Services eclecTA. Women's Health and Education Center (WHEC) - Obstetrics - Intrapartum Electronic Fetal Heart Rate Monitoring [Internet]. Women's Health and Education Center | WHEC |. [cited 2018Sep10]. Available from: http://womenshealthsection.com/content/obs/obs028.php3

[2] Abramowicz JS. Benefits and risks of ultrasound in pregnancy. Seminars in Perinatology. 2013;37(5):295-300.

[3] Cesarelli M, Ruffo M, Romano M, Bifulco P. Simulation of foetal phonocardiographic recordings for testing of FHR extraction algorithms. Computer Methods and Programs in Biomedicine. 2012;107(3):513-23.

[4] Koutsiana E, Hadjileontiadis LJ, Chouvarda I, Khandoker AH. Fetal heart sounds detection using wavelet transform and fractal dimension. Frontiers in Bioengineering and Biotechnology. 2017;5.

[5] Kumar D, Carvalho P, Antunes M, Henriques J, Maldonado M, Schmidt R, Habetha J. Wavelet transform and simplicity based heart murmur segmentation. Computers in Cardiology. 2006;13:173-6.

[6] Vepa J, Tolay P, Jain A. Segmentation of heart sounds using simplicity features and timing information. 2008 IEEE International Conference on Acoustics, Speech and Signal Processing. 2008;469-472.

[7] Korven J. A comparison of wavelet and simplicity-based heart sound and murmur segmentation methods. [thesis]. San Luis Obispo: Faculty of California Polytechnic State University;2006.

[8] Hadjileontiadis L. Wavelet-based enhancement of lung and bowel sounds using fractal dimension thresholding — part I: methodology. IEEE Transactions on Biomedical Engineering. 2005;52(6):1143-8.

[9] Ibrahim EA, Awar SA, Balayah ZH, Hadjileontiadis LJ, Khandoker AH. A comparative study on fetal heart rates estimated from fetal phonography and cardiotocography. Frontiers in Physiology. 2017;8.

Address for correspondence.

Elisavet Koutsiana, koutsiana@auth.gr

Laboratory of Medical Informatics, the Medical School, Aristotle University, GR 54124 Thessaloniki, Greece 